\title{
Long-lasting effects of human mesenchymal stem cell systemic administration on pain-like behaviors, cellular, and biomolecular modifications in neuropathic mice
}

\author{
Dario Siniscalco ${ }^{1}$ *, Catia Giordano ${ }^{1}$, Umberto Galderisi ${ }^{2}$, Livio Luongo ${ }^{1}$, Vito de Novellis ${ }^{1}$, Francesco Rossi ${ }^{1}$ \\ and Sabatino Maione ${ }^{1 *}$
}

1 Division of Pharmacology "L. Donatelli," Department of Experimental Medicine, Second University of Naples, Naples, Italy

2 Division of Biotechnology and Molecular Biology "A. Cascino," Second University of Naples, Naples, Italy

Edited by:

Hongyun Huang, Neuroscience Institute of Taishan Medical

University, China

\section{Reviewed by:}

Russell J. Andrews, NASA Ames Research Center, USA

Lin Chen, Beijing Hongtianji

Neuroscience Academy, China

${ }^{*}$ Correspondence:

Dario Siniscalco and Sabatino Maione, Division of Pharmacology,

Department of Experimental

Medicine, Second University of

Naples, via S. Maria di Costantinopoli,

16, 80138 Napoli, Italy.

e-mail:dariosin@uab.edu;

sabatino.maione@unina2.it
Background: Neuropathic pain (NP) is an incurable disease caused by a primary lesion in the nervous system. NP is a progressive nervous system disease that results from poorly defined neurophysiological and neurochemical changes. Its treatment is very difficult. Current available therapeutic drugs have a generalized nature, sometime acting only on the temporal pain properties rather than targeting the several mechanisms underlying the generation and propagation of pain. Methods: Using biomolecular and immunohistochemical methods, we investigated the effect of the systemic injection of human mesenchymal stem cells (hMSCs) on NP relief. We used the spared nerve injury (SNI) model of NP in the mouse. hMSCs were injected into the tail vein of the mouse. Stem cell injection was performed 4 days after sciatic nerve surgery. Neuropathic mice were monitored every 10 days starting from day 11 until 90 days after surgery. Results: hMSCs were able to reduce pain-like behaviors, such as mechanical allodynia and thermal hyperalgesia, once injected into the tail vein. An anti-nociceptive effect was detectable from day 11 post surgery ( 7 days post cell injection). hMSCs were mainly able to home in the spinal cord and pre-frontal cortex of neuropathic mice. Injected hMSCs reduced the protein levels of the mouse pro-inflammatory interleukin IL-1 $\beta$ and IL-17 and increased protein levels of the mouse anti-inflammatory interleukin IL-10, and the marker of alternatively activated macrophages CD106 in the spinal cord of SNI mice. Conclusion: As a potential mechanism of action of hMSCs in reducing pain, we suggest that they could exert their beneficial action through a restorative mechanism involving: (i) a cell-to-cell contact activation mechanism, through which spinal cord homed hMSCs are responsible for switching pro-inflammatory macrophages to anti-inflammatory macrophages; (ii) secretion of a broad spectrum of molecules to communicate with other cell types. This study could provide novel findings in MSC pre-clinical biology and their therapeutic potential in regenerative medicine.

Keywords: neuropathic pain, cell transplantation, human mesenchymal stem cells, macrophages

\section{INTRODUCTION}

Chronic pain, defined as pain that extends beyond the expected period of healing (Merskey and Bogduk, 1994), can be classified as nociceptive pain and neuropathic pain (NP): the first one derives from direct activation of peripheral nociceptors following a tissue damage and it is associated with inflammation. NP is a progressive nervous system disease, occurring in association with a primary lesion or dysfunction in the nervous system. It could be often associated with a continuous burning character together with the presence of abnormal sensory symptoms, such as hyperalgesia (an increased response to a stimulus which is normally painful; patients with hyperalgesia perceive pain spontaneously) and allodynia (pain as a result of a stimulus which does not provoke pain; patients with allodynia do not feel constant pain; indeed in the absence of a stimulus there is no pain; Merskey and Bogduk, 1994; Siniscalco et al., 2005a). NP is difficult to treat and often unresponsive to all available therapies. The array of drugs available today in the treatment of NP indicates a heavy reliance on "second-indication" drugs, which were initially developed to treat other primary indications (e.g., anticonvulsants, antidepressants; Galluzi, 2005; Siniscalco et al., 2005b).

Nowadays, stem cell therapy represents the greatest promise for the future of molecular medicine (Siniscalco et al., 2007). Stem cells could be neuroprotective in a variety of nervous system injury models (Cao et al., 2002; Lindvall and Kokaia, 2005, 2006). Similar to neurodegenerative diseases, NP appears to be amenable to stem cell therapy (Klass et al., 2007; Siniscalco, 2010).

Stem cell populations comprise several kinds of undifferentiated cells; among these, mesenchymal stem cells (MSCs) are believed to have the best potential results in pain-care research (Klass et al., 2007). Indeed, the ease of human mesenchymal stem cell (hMSC) isolation from adult tissues and the fact they are not ethically restricted make them attractive potential candidates for use in cell therapy. In addition, their strong immunosuppressive 
properties render them useful for transplantations, without requiring a pharmacological immunosuppression (Le Blanc and Pittenger, 2005; Beggs et al., 2006; Petrie Aronin and Tuan, 2010). Their unique anti-inflammatory immunomodulatory phenotype is another valuable property that can be used in diseases such as NP, where a controlled inflammatory response is required. These cells are a select population of progenitor cells of mesodermal origin found in the adult bone marrow, but can also be found in the perivascular regions of most tissues, such as adipose, skeletal muscle, and the umbilical cord (Zuk et al., 2002; Sarugaser et al., 2005). MSCs demonstrate an ability to differentiate along several tissue-specific lineages, a high expansion potential, genetic stability, stable phenotype, and a self-renewing capacity (Giordano et al., 2007; Pricola et al., 2009).

In NP models, MSCs have been observed to modulate pain behavior after sciatic nerve injury (Musolino et al., 2007), although their exact mechanism of action on pain behavior requires further clarification. Moreover, we already demonstrated that hMSCs were able to reduce short-term pain-like behaviors and modify painrelated molecular mechanisms once transplanted into the cerebral ventricle of neuropathic mice (Siniscalco et al., 2010).

However in the present study, we have used the spared nerve injury (SNI) mouse model of NP to investigate the effects of systemic intravenous administration of hMSCs on long-term painassociated behavior and on cellular and molecular mechanisms underlying the development and maintenance of NP.

\section{MATERIALS AND METHODS ANIMALS}

Male CD-1 mice (35-40 g) were housed three per cage under controlled illumination (12:12 h light:dark cycle; light on $06.00 \mathrm{~h})$ and environmental conditions (room temperature $20-22^{\circ} \mathrm{C}$, humidity 55-60\%) for at least 1 week before the commencement of experiments. Mouse chow and tap water were available ad libitum. The experimental procedures were approved by the Ethic Committee of the Second University of Naples. Animal care was in compliance with the IASP and European Community (E.C. L358/1 18/12/86) guidelines on the use and protection of animals in experimental research. All efforts were made to minimize animal suffering and to reduce the number of animals used. Behavioral testing was performed before surgery to establish a baseline for comparison with post-surgical values. Mononeuropathy was induced according to the method of Bourquin and Decosterd (Bourquin et al., 2006). Mice were anesthetized with sodium pentobarbital $(60 \mathrm{mg} / \mathrm{kg}$ i.p.). The right hindlimb was immobilized in a lateral position and slightly elevated. Incision was made at mid-thigh level using the femur as a landmark. The sciatic nerve was exposed at mid-thigh level distal to the trifurcation and freed of connective tissue; the three peripheral branches (sural, common peroneal, and tibial nerves) of the sciatic nerve were exposed without stretching nerve structures. Both tibial and common peroneal nerves were ligated and transacted together. A micro-surgical forceps with curved tips was delicately placed below the tibial and common peroneal nerves to slide the thread ( 5.0 silk, Ethicon, Johnson, and Johnson Intl, Brussels, Belgium) around the nerves. A tight ligation of both nerves was performed. The sural nerve was carefully preserved by avoiding any nerve stretch or nerve contact with surgical tools. Muscle and skin were closed in two distinct layers with silk 5.0 suture. Intense, reproducible and long-lasting thermal hyperalgesia and mechanical allodynia-like behaviors are measurable in the non-injured sural nerve skin territory (Bourquin et al., 2006). The SNI model offers the advantage of a distinct anatomical distribution with an absence of co-mingling of injured and non-injured nerve fibers distal to the lesion such as the injured and non-injured nerves and territories can be readily identified and manipulated for further analysis (i.e., behavioral assessment).

The sham procedure consisted of the same surgery without ligation and transection of the nerves.

Groups of mice were divided as follows:

(i) Naïve $(n=9)$;

(ii) Sham-operated mice $(n=9)$;

(iii) SNI mice treated with vehicle (SNI/vehicle; $n=18$ );

(iv) SNI mice treated with hMSCs $\left(2 \times 10^{6}\right.$ cells $/ 100 \mu \mathrm{l}$; SNI/hMSCs; $n=18$ ).

On day 30 after surgery, mice were divided into further groups: (a) groups for RT-PCR examination (three naive mice, three sham mice, six SNI/vehicle mice, and six SNI/hMSCs mice), (b) groups for western blot examination (three naive mice, three sham mice, six SNI/vehicle mice, and six SNI/hMSCs mice), (c) groups for histological examination (three naive mice, three sham mice, six SNI/vehicle mice, and six SNI/hMSCs mice).

\section{NOCICEPTIVE BEHAVIOR}

Thermal hyperalgesia was evaluated by using a Plantar Test Apparatus (Ugo Basile, Varese, Italy). On the day of the experiment each animal was placed in a plastic cage $(22 \mathrm{~cm} \times 17 \mathrm{~cm} \times 14 \mathrm{~cm}$; length $\times$ width $\times$ height) with a glass floor. After a 60-min habituation period, the plantar surface of the hind paw was exposed to a beam of radiant heat through the glass floor. The radiant heat source consisted of an infrared bulb (Osram halogen-bellaphot bulb; $8 \mathrm{~V}, 50 \mathrm{~W}$ ). A photoelectric cell detected light reflected from the paw and turned off the lamp when paw movement interrupted the reflected light. The paw withdrawal latency (PWL) was automatically displayed to the nearest $0.1 \mathrm{~s}$; the cut-off time was $20 \mathrm{~s}$ in order to prevent tissue damage.

Mechanical allodynia was measured by using Dynamic Plantar Anesthesiometer (Ugo Basile, Varese, Italy). Mice were allowed to move freely in one of the two compartments of the enclosure positioned on the metal mesh surface. Mice were adapted to the testing environment before any measurements were taken. After that, the mechanical stimulus was delivered to the plantar surface of the hindpaw of the mouse from below the floor of the test chamber by an automated testing device. A steel rod $(2 \mathrm{~mm})$ was pushed with electronical ascending force $(0-30 \mathrm{~g}$ in $10 \mathrm{~s})$. When the animal withdrawn its hindpaw, the mechanical stimulus was automatically withdrawn and the force recorded to the nearest $0.1 \mathrm{~g}$. Nociceptive responses for thermal and mechanical sensitivity were expressed as thermal PWL in seconds and mechanical paw withdrawal threshold (PWT) in grams.

Each mouse served as its own control, the responses being measured both before and after surgical procedures. PWL and PWT were quantified by an observer blinded to the treatment. 


\section{MOTOR COORDINATION BEHAVIOR}

Neurological functions and motor coordination were evaluated by Rotarod motor test (Ugo Basile, Varese, Italy). This test consists of putting the mouse on a rotary cylinder in order to then measure the time (in seconds) of its equilibrium before falling. The cylinder is subdivided in five sections, allowing the screening of five animals per test (one for section), simultaneously. Below the cylinder there is a platform in its turn subdivided in five plates (in correspondence of the five sections) everyone of which it is connected to a magnet that, activated from the fall of the mouse on the plate, records the time of permanence on the cylinder. After a period of adaptation of $30 \mathrm{~s}$, the spin speed gradually increased from 5 to $40 \mathrm{~g}$ for the maximum time of $5 \mathrm{~min}$. On the same day, the animals were analyzed by two separate tests with a time interval of $1 \mathrm{~h}$. The experiment was performed for every group of animals: the day before the surgical procedure, the day before the behavioral tests in order to avoid useless stress and the day before the stem cell administration. The time of permanence of the mouse on the cylinder was expressed as latency(s).

\section{HUMAN MESENCHYMAL STEM CELL CULTURES}

Human bone marrow derived MSCs were isolated from a small aspirate of bone marrow and in vitro expanded in basic-fibroblast growth factor (FGF)-containing medium as already described (Siniscalco, 2010; Siniscalco et al., 2010). Shortly, bone marrows were obtained from healthy donors after informed consent. Bone marrow aspirates were collected from iliac crests, separated on Ficoll density gradient (GE Healthcare, Italy), and the mononuclear cell fraction was harvested and washed in phosphate buffer saline (PBS; Sigma, St. Louis, MO, USA). $1-2.5 * 10^{5}$ cells $/ \mathrm{cm}^{2}$ were seeded in $100 \mathrm{~mm}$ dishes with $\alpha$-modified Eagle's medium ( $\alpha$-MEM; Lonza, Verviers, Belgium) containing 10\% fetal bovine serum (FBS; EuroClone, Celbio, Milan, Italy), $2 \mathrm{ng} / \mathrm{ml}$ basic FGF (PeproTech, Rocky Hill, NJ, USA), 2 mM L-glutamine (Lonza, Verviers, Belgium), $100 \mathrm{U} / \mathrm{ml}$ penicillin (Lonza, Verviers, Belgium), and $100 \mathrm{mg} / \mathrm{ml}$ streptomycin (Lonza, Verviers, Belgium; proliferating medium). After $24-48 \mathrm{~h}$, non-adherent cells were discarded, and adherent cells representing MSCs along with committed progenitors were washed twice with PBS. Cells were then incubated for 7-10 days in proliferating medium to reach confluence and extensively propagated for further experiments. We used cells till the sixth passage, each time we plated $2 \times 10^{3}$ cells $/ \mathrm{cm}^{2}$.

We routinely characterized hMSCs by staining for the mesenchymal stem cell cyto-specific markers CD73, CD90, and CD105, as already described (Siniscalco, 2010; Siniscalco et al., 2010).

\section{HUMAN MESENCHYMAL STEM CELL TRANSPLANTATION PROCEDURE}

On the day of the administration, hMSCs were microinjected into the tail vein by using an $1-\mathrm{ml}$ syringe. A volume of $100 \mu \mathrm{l}$ solution (stem cells + vehicle) or vehicle were injected. hMSCs were suspended in PBS with 10\% heparin and transplanted to neuropathic mice, while control mice were administered with $100 \mu \mathrm{l}$ PBS with $10 \%$ heparin (vehicle solution). Immediately prior to administration, hMSCs were maintained at room temperature, and they were gently re-suspended with a pipette to ensure they were not aggregated before infusion. We used heparin in the injection solution in order to avoid cell cluster formation (Siniscalco et al., 2010). Each neuropathic mice received $2 \times 10^{6}$ cells $/ 100 \mu$ l. The amount of cells to be injected was chosen on the basis of a previous study (Lee et al., 2009). In addiction, it has been observed that a higher number of cells may cause cell cluster formation within the microinjection cannula which, in turn, may be responsible of cell damaging and decreased viability. After the administration, antibiotic was applied to the injection area to prevent infection. Immunosuppressants were not used in any animal.

Stem cells injection was performed 4 days after sciatic nerve surgery. Neuropathic mice were monitored every 10 days starting from day 11 until 90 days after surgery.

\section{RNA EXTRACTION AND RT-PCR}

Total RNA was extracted from homogenized mouse sciatic nerve, lung, lumbar L4-L5 spinal cord (from ventral and dorsal areas, respectively), and pre-frontal cortex using an RNA Tri-Reagent (Molecular Research Center Inc., Cincinnati, OH, USA) according to the manufacturer's protocol. The total RNA concentration was determined by UV spectrophotometer. The mRNA levels of the glyceraldehyde phosphate dehydrogenase $(G A P D H)$ gene under analysis were measured by RT-PCR amplification, as previously reported (Siniscalco et al., 2010). Reverse transcriptase from avian myeloblastosis virus (AMV-RT; Promega, Madison, WI, USA) was used. Briefly, for first-strand cDNA synthesis $100 \mathrm{ng}$ total RNA, random examers, dNTPs (Promega, Madison, WI, USA), AMV buffer, AMV-RT, and recombinant RNasin ribonuclease inhibitor (Promega, Madison, WI, USA) were assembled in diethyl-pyrocarbonate-treated water to a $20-\mu$ l final volume and incubated $10 \mathrm{~min}$ at $65^{\circ} \mathrm{C}$ and $1 \mathrm{~h}$ at $42^{\circ} \mathrm{C}$. Aliquots of $3 \mu \mathrm{l}$ cDNA were transferred into a $25-\mu \mathrm{l}$ PCR reaction mixture containing dNTPs, $\mathrm{MgCl}_{2}$ buffer, specific primers, and GoTaq Flexi DNA polymerase (Promega, Madison, WI, USA) and amplification reactions using specific primers and conditions for human GAPDH cDNA were carried out. RT minus controls were carried out to check potential genomic DNA contamination. These RT minus controls were performed without using the reverse transcriptase enzyme in the reaction mix. Sequence for the human mRNA from GenBank (DNASTAR Inc., Madison, WI, USA) was used to design specific primer pairs for RT-PCRs (OLIGO 4.05 software, National Biosciences Inc., Plymouth, MN, USA; primers available on request). Each RT-PCR was repeated at least three times to achieve best reproducibility data. The mean of the interassay variability of each RT-PCR assays was 0.07 . PCR products were resolved into $2.0 \%$ agarose gel.

\section{PROTEIN EXTRACTION AND WESTERN BLOT ANALYSIS}

For the protein extraction, the lumbar L4-L5 segment of spinal cord was minced into small pieces with a blender on ice, then was suspended in lysis buffer (Tris/ $\mathrm{HCl} 50 \mathrm{mM}, \mathrm{pH} 7.4$; $\mathrm{NaCl} 250 \mathrm{mM}$; 0.1\% Triton X-100; Protease Inhibitor Cocktail (Roche, Mannheim, Germany); $\mathrm{NaF} 0.05 \mathrm{M} ; \mathrm{Na}_{3} \mathrm{VO}_{4} 0.1 \mathrm{mM}$; PMSF $100 \mu \mathrm{g} / \mathrm{ml}$ ) containing $6 \mathrm{M}$ urea. The protein concentration was determined by the method described by Bradford (1976). Each sample was loaded, electrophoresed in a $15 \%$ SDSpolyacrylamide gel ( $8 \%$ for CD206 detection) and electroblotted onto a nitrocellulose membrane. The membrane was blocked in 
$5 \%$ milk, $1 \times$ Tris-buffered saline, $0.05 \%$ Tween-20. Primary antibodies (Santa Cruz Biotechnology, Santa Cruz, CA, USA) to detect IL-1 $\beta$, IL-17, IL-10, and CD106 were used according to the manufacturer at 1:500 dilutions. Immunoreactive signals were detected with a horseradish peroxidase-conjugated secondary antibody and reacted with an ECL system (Amersham Pharmacia, Uppsala, Sweden). Protein levels were normalized with respect to the signal obtained with anti- $\beta$-tubulin monoclonal antibodies (Santa Cruz Biotechnology, Santa Cruz, CA, USA; 1:1000 dilution). The semiquantitative analysis of protein levels was carried out by the "Gel Doc 2000 UV System" (Bio-Rad, Hercules, CA, USA).

\section{FLUORESCENCE IMMUNOHISTOCHEMISTRY}

Animals were perfused transcardially with $150 \mathrm{ml}$ of saline solution $(0.9 \% \mathrm{NaCl})$, and $250 \mathrm{ml}$ of $4 \%$ paraformaldehyde fixative. The L4-L5 spinal cord was taken out and kept in the fixative for $24 \mathrm{~h}$ at $4^{\circ} \mathrm{C}$. The tissue was kept in $30 \%$ sucrose in PBS and frozen in cryostat embedding medium (Bio-Optica, Milan, Italy). Serial $15 \mu \mathrm{m}$ sections of the L4-L5 spinal cord were cut using a cryostat and thaw-mounted onto glass slides. After washing in PBS, non-specific antibody binding was inhibited by incubation for $30 \mathrm{~min}$ in blocking solution (1\% BSA, $0.2 \%$ powdered skim milk, $0.3 \%$ Triton X-100 in PBS). Primary antibodies were diluted in PBS blocking buffer and slides were incubated overnight at $4{ }^{\circ} \mathrm{C}$ in primary antibodies to mouse monoclonal anti-CD73 (1:200; Abcam, Cambridge, UK), to goat monoclonal anti-interleukin $1 \beta$ (IL-1 $\beta, 1: 200$; Santa Cruz Biotech, CA, USA), to rat polyclonal anti-interleukin 17 (IL-17, 1:200; Santa Cruz Biotech, CA, USA), to mouse monoclonal anti-CD4 (1:250; Santa Cruz Biotech, CA, USA), to rabbit poly-clonal anti-Glial Fibrillary Acid Protein (GFAP, 1:1000; Dako Cytomation, Denmark), and to rabbit poly-clonal anti-ionized calcium binding adapter molecule1 (Iba1, 1:500; Wako Chemicals, Japan). Fluorescent-labeled secondary antibodies (1:500; Alexa Fluor 488, 568, Molecular Probe, Invitrogen, Carlsbad, CA, USA) specific to the IgG species used as a primary antibody were used to locate the specific antigens in each section. Sections were mounted with Vectashield containing DAPI mounting medium (Vector Laboratories, Burlingame, CA, USA). Fluorescently labeled sections were viewed with a fluorescence microscope (Leica, Wetzlar, Germany) to locate the cells and identify the spinal cord area. Immunofluorescence images of four stained sections were taken for each animal.

\section{STATISTICAL ANALYSIS}

Behavioral and molecular data are shown as mean \pm SEM. ANOVA, followed by Student-Neuman-Keuls post hoc test, was used to determine the statistical significance among groups. $p<0.05$ was considered statistically significant.

\section{RESULTS \\ HUMAN MESENCHYMAL STEM CELL ADMINISTRATION IN NEUROPATHIC MICE REDUCES MECHANICAL ALLODYNIA, THERMAL HYPERALGESIA, AND DOES NOT AFFECT MOTOR FUNCTION}

Neuropathic mice were subjected to intravenous stem cell administration 4 days after sciatic nerve injury and were monitored every 10 days starting from day 11 until 90 days post surgery. We injected hMSCs when NP was already established (4 days after SNI surgery) in order to assess their potential role as a therapeutic tool in long-term pain care. hMSCs were able to reduce pain-like behaviors such as mechanical allodynia and thermal hyperalgesia once intravenously injected (Figures 1A,B).

Nociceptive responses to thermal and mechanical stimuli (PWL and PWT, respectively) were measured every $30 \mathrm{~min}$ for $3 \mathrm{~h}$ before surgery or every 10 days starting from day 11 until 90 days after sciatic nerve surgery. The mean PWL and PWT was calculated from six consecutive trials (each performed every $30 \mathrm{~min}$ ) and averaged for each group of mice.

Sham mice treated with vehicle did not show any change in PWL or PWT compared to the naïve mice (data not shown). SNI mice treated with vehicle developed mechanical allodynia and thermal hyperalgesia on the ipsilateral side at all time points that we analyzed, compared to sham mice treated with vehicle (Figures 1A,B), although not on the contralateral side (data not shown). Administration of hMSCs into the tail vein of SNI mice significantly reduced mechanical allodynia and thermal hyperalgesia on the ipsilateral side (Figures 1A,B) without any change in the contralateral side (data not show) compared to SNI mice treated with vehicle.

Stem cell administration did not affect the neurological functions of the neuropathic mice, once systematically injected (Figure 1C). Neither the administration procedure nor stem cells modified motor coordination. The pain model we used involves a lesion of two of the three terminal branches of the sciatic nerve (tibial and common peroneal nerves, leaving the remaining sural nerve intact), in which neuropathic mice proved to be less able to walk respect to naïve mice. In order to exclude any further motor impairment that could possibly be generated by hMSCs, we applied the Rotarod test to compare SNI/hMSC with SNI/vehicle treated mice. As shown in Figure 1C, there is no difference in the motor coordination outcome for these two groups of animals.

\section{HUMAN MSCS HOME NEIGHBORING TO THE NP CONTROLLING AND PROCESSING SITES AFTER ADMINISTRATION}

In order to verify in which tissue the intravenously injected hMSCs were able to home, we performed specific RT-PCR amplification of human GAPDH gene. The sciatic nerve, lung, dorsal, and ventral L4-L5 lumbar spinal cord and pre-frontal cortex were harvested from hMSC-injected neuropathic mice 30 days after SNI injury (26 days after stem cell injection) to verify the homing of hMSCs at these sites. Biomolecular analysis conducted on RNA extracted from these sites revealed a preferential accumulation of hMSCs in the L4-L5 spinal cord and pre-frontal cortex (Figure 2A). A very weak band was found in the lung. No GAPDH band was detectable in the sciatic nerve, thus revealing the specificity of hMSC engraftment in the NP controlling and processing areas (Figure 2A). The primers we used to perform RT-PCR analysis were designed to be specifically direct to the human $G A P D H$ gene. However, in order to confirm hMSC homing in the dorsal and ventral L4-L5 spinal cord of neuropathic mice, we successfully verified that injected hMSCs expressed the surface antigens CD73 (NT5E, ecto-5' -nucleotidase, E5NT, NTE) using immunocytochemistry (Figures 2B-D). The expression of these antigens is a valid marker recognized as one of the criteria in the identification of MSCs (Siniscalco et al., 2010). The mouse anti-human CD73 


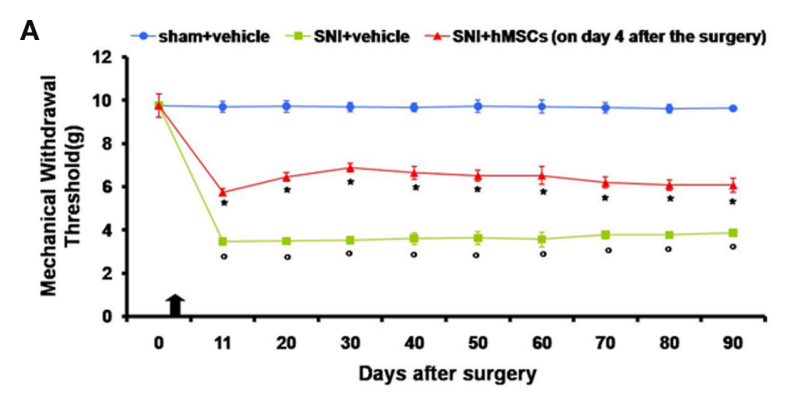

B
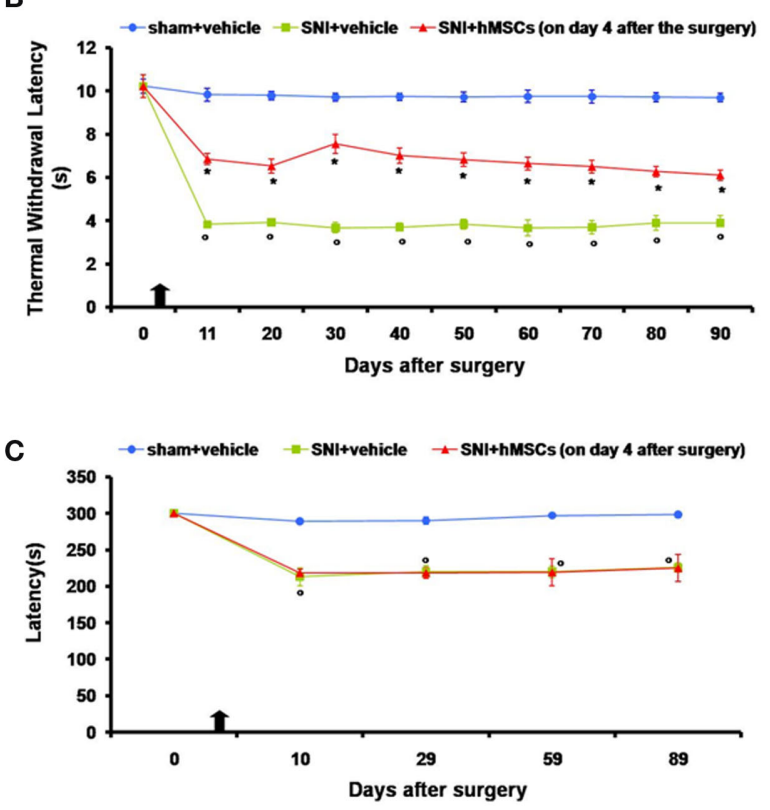

FIGURE 1 | (A) Effects of human mesenchymal stem cells on reflex withdrawal responses ( $g$, mean \pm SEM) to mechanical noxious stimuli in SNI mice. The onset of SNI-induced mechanical allodynia was evaluated in ipsilateral sides every 10 days starting from day 11 until 90 days after surgery. Mice showed a significant reduction in the threshold to mechanical stimulation in the ipsilateral paw ( ${ }^{*} p<0.05$ vs. sham-operated mice) after SNI surgery. Human MSC treatment (on day 4 after SNI surgery, as indicated by the arrow) prevented the appearance of mechanical allodynia at $11,20,30,40,50,60,70,80$, and 90 days post-SNI ( $p<0.05$ vs. SNI mice). (B) Effects of human mesenchymal stem cells on reflex withdrawal responses ( $s$, mean \pm SEM) to thermal noxious stimuli in SNI mice. The onset of SNI-induced thermal hyperalgesia was evaluated in ipsilateral sides every 10 days starting from day 11 until 90 days after surgery. SNI mice showed a significant reduction in withdrawal latency to radiant heat in the ipsilateral paw ( ${ }^{*} p<0.05$ vs. sham-operated mice). Human MSC treatment (on day 4 after SNI surgery, as indicated by the arrow) prevented the appearance of thermal hyperalgesia $11,20,30,40,50,60,70,80$, and 90 days post-SNI ( ${ }^{\circ} p<0.05$ vs. SNI mice). (C) Rotarod motor testing results. The effects of human mesenchymal stem cell injection on motor performance in the Rotarod test is shown. Systemic administration of hMSCs in tail vein of SNI mice had no effect on motor function compared with vehicle treated SNI mice. Human MSCs were injected on day 4 after the SNI surgery. Results are expressed as the mean \pm SEM of the latency (s; $n=5$ mice/group). ${ }^{*} p<0.05$ vs. sham/vehicle.

antibody detects glycosyl phosphatidylinositol (GPI)-anchored purine salvage enzyme expressed on the surface of hMSCs. The antibody does not cross-react with other human antigens or with

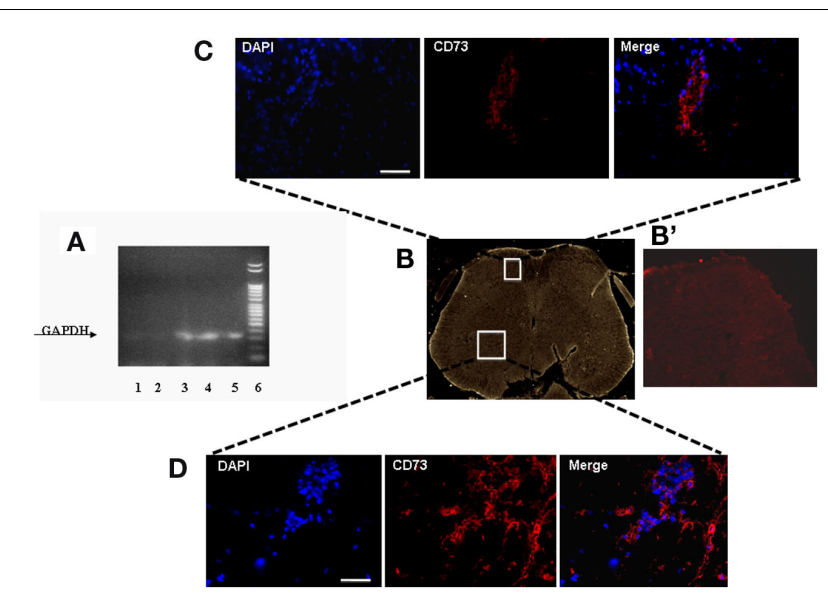

FIGURE 2 | (A) Representative agarose gel blot analysis for human GAPDH gene of 30 days-SNI/hMSCs mice following RT-PCR is shown. Lane (1) lung; lane (2) sciatic nerve; lane (3) ventral L4-L5 spinal cord and (4) dorsal L4-L5 spinal cord; lane (5) pre-frontal cortex; lane (6) DNA ladder marker. The analysis of mRNA levels was carried out by the "Gel Doc 2000 UV System" (Bio-Rad, Hercules, CA, USA). RT-PCR analysis revealed a preferential accumulation of hMSCs to the L4-L5 spinal cord (both ventral and dorsal areas) and pre-frontal cortex. Very weak band was found from lung. No GAPDH band was detectable in sciatic nerve, thus revealing the specificity of hMSC engraftment at the NP controlling and processing areas. (B) Representative cross-section of mouse whole L4-L5 spinal cord area from hMSC-treated 30 days-neuropathic mice (26 days post-hMSC injection). (B') Representative cross-section of mouse whole L4-L5 spinal cord area from vehicle treated 30 days-neuropathic mice showing no staining for human specific CD73. (C,D) Human MSCs expressed lineage-specific antigens at 26 days post-injection in vivo. Representative fluorescent photomicrograph of hMSCs showing immunocytochemistry for CD73. Area in white rectangle insets is shown: (C) ventral spinal cord; (D) dorsal spinal cord; left: cell nuclei were counterstained with DAPI (blue fluorescence); middle: CD73-positive hMSCs emitted red fluorescence; right: pictures with both red and blue fluorescence were merged. Scale bars: $100 \mu \mathrm{m}$.

other mouse (host) antigens. Injected hMSCs were positive for these lineage-specific antigens 26 days after the administration procedure.

\section{HUMAN MESENCHYMAL STEM CELLS REDUCE IL-1 $\beta$ AND IL-17 PRO-INFLAMMATORY PROTEIN LEVELS AND INCREASE IL-10 ANTI-INFLAMMATORY PROTEIN LEVELS IN NEUROPATHIC MICE}

To verify an anti-inflammatory or immunomodulatory action for stem cell-mediated anti-allodynic/anti-hyperalgesic mechanisms we checked inflammatory interleukin levels by western blot analysis 30 days after sciatic nerve surgery. We chose this time point on the basis of behavioral results, indicating a slight maximum anti-nociceptive action taken by hMSCs at this time.

Human MSCs were able to reduce the pro-inflammatory interleukin IL-1 $\beta$ and IL-17 protein levels in the lumbar L4-L5 spinal cord of SNI/hMSC-treated mice compared to SNI/vehicle mice (Figure 3). Conversely, hMSCs did trigger antiinflammatory interleukin IL-10 over-production in the spinal cord of SNI/hMSCs-treated mice compared to SNI/vehicle mice (Figure 3).

For all proteins examined, naïve and sham animals showed no differences in the expression levels (data not shown). 


\section{Immunoblot (IB) analysis of IL-1 $\beta$, IL-17, IL-10, CD206 protein levels}
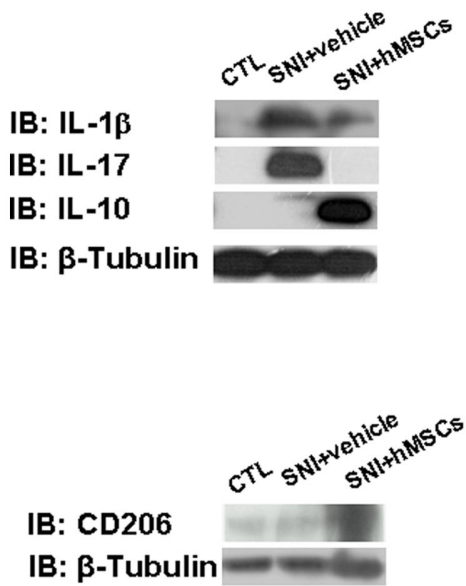

FIGURE 3 | Representative western blot analysis for IL-1 $\beta$, IL-17, IL-10, CD206, and housekeeping $\boldsymbol{\beta}$-tubulin mouse proteins are shown. The semi-quantitative analysis of protein levels was carried out by the "Gel Doc 2000 UV System" (Bio-Rad, Hercules, CA, USA). Human MSC treatment (on day 4 after SNI surgery) reduced the protein levels of pro-inflammatory interleukins IL-1 $\beta$ and IL-17 in hMSC-treated 30 days-neuropathic mice (SNI + hMSCs) respect to vehicle treated 30 days-neuropathic mice (SNI + vehicle). Human MSC treatment (on day 4 after SNI surgery) increased the protein levels of anti-inflammatory interleukin IL-10 and activated anti-inflammatory macrophage marker CD206 in hMSC-treated 30 days-neuropathic mice (SNI + hMSCs) respect to vehicle treated 30 days-neuropathic mice (SNI + vehicle). Upper graph shows IL-1 $\beta / \beta$-tubulin normalized values as obtained by immunoblot analysis. Human MSC
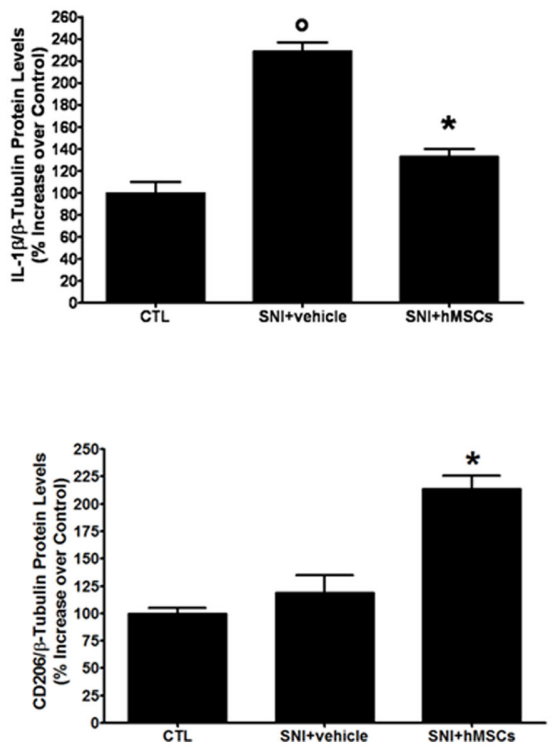

treatment (on day 4 after SNI surgery) reduced the protein levels of $I L-1 \beta$ interleukin in hMSC-treated 30 days-neuropathic mice (SNI + hMSCs) respect to vehicle treated 30 days-neuropathic mice (SNI + vehicle). ANOVA, followed by Student-Neuman-Keuls post hoc test, was used to determine the statistical significance among groups. ${ }^{*} p<0.01$ was considered statistically significant. Lower graph shows CD206/ $\beta$-tubulin normalized values as obtained by immunoblot analysis. Human MSC treatment (on day 4 after SNI surgery) increased the protein levels of activated anti-inflammatory macrophage marker CD206 in hMSC-treated 30 days-neuropathic mice (SNI+hMSCs) respect to vehicle treated 30 days-neuropathic mice (SNI + vehicle). ANOVA, followed by Student-Neuman-Keuls post hoc test, was used to determine the statistical significance among groups. ${ }^{*} p<0.01$ was considered statistically significant.

\section{HUMAN MESENCHYMAL STEM CELLS INCREASE CD206 MACROPHAGE} MARKER IN THE SPINAL CORD OF SNI MICE

To test the hypothesis that hMSCs may induce an antiinflammatory phenotype in macrophages, we examined levels of CD206 protein, a well-known marker for anti-inflammatory macrophages (Porcheray et al., 2005), by western blot analysis 30 days after sciatic nerve surgery.

Indeed, hMSCs were able to increase the CD206 levels in the L4-L5 spinal cord of SNI/hMSC-treated mice compared to $\mathrm{SNI} /$ vehicle mice (Figure 3).

\section{T LYMPHOCYTES AND ASTROCYTES ARE RESPONSIBLE RESPECTIVELY FOR PRO-INFLAMMATORY IL-17 AND IL-1 $\beta$ PRODUCTION IN THE SPINAL CORD OF SNI MICE}

As described above, hMSCs were able to decrease the levels of pro-inflammatory interleukin IL-17 protein in the lumbar L4-L5 spinal cord of SNI/hMSC-treated mice compared to SNI/vehicle mice. To verify immunomodulatory action mediated by hMSCs further, we double stained for IL-17 and for the T lymphocyte marker CD4. IL-17 positive profiles were found co-localized with
CD4 T lymphocyte marker positive profiles in the L4-L5 spinal cord of 30 days NP mice (Figure 4).

Human MSCs were able to reduce the levels of proinflammatory interleukin IL-1 $\beta$ in the L4-L5 spinal cord of $\mathrm{SNI} /$ hMSC-treated mice compared to SNI/vehicle mice. In order to verify which cells were responsible for the pro-inflammatory production of IL-1 $\beta$, we double stained for IL- $1 \beta$ and GFAP and Iba-1 cyto-specific markers, respectively. IL-1 $\beta$ positive profiles were found co-localized with GFAP astrocytic marker positive profiles (Figure 4), but not with specific Iba-1 microglial marker (data not shown) in the L4-L5 spinal cord of 30 days NP mice.

\section{DISCUSSION}

In this study we demonstrated for the first time that a single systemic injection of hMSCs into the tail vein was able to reduce NP-like behaviors and to modify molecular and cellular pain-associated pathways.

We chose the tail vein as the injection site for systemic delivery of hMSCs since the present study focused on (i) further clarification of the beneficial effects of stem cells in NP, without any 


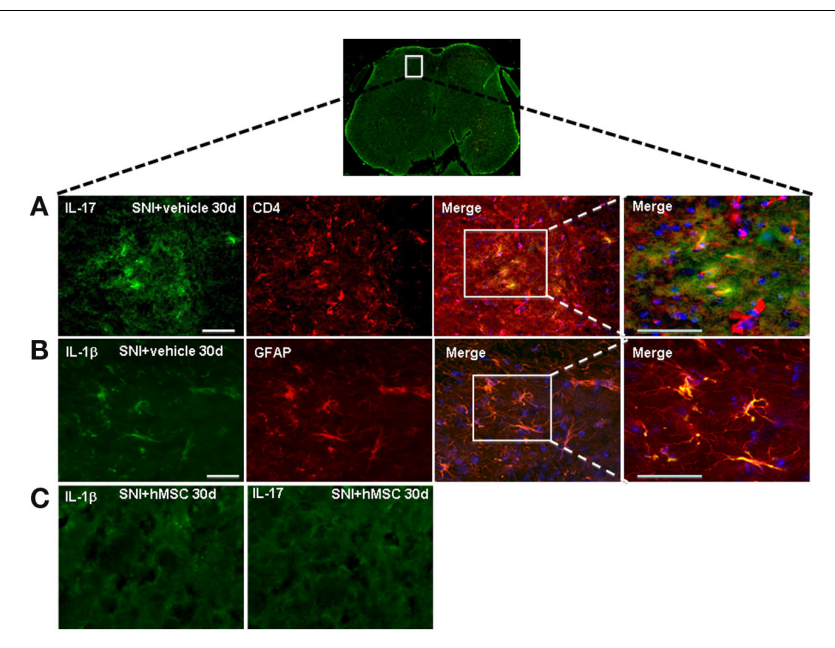

FIGURE 4 | Representative cross-section of mouse L4-L5 spinal cord from $\mathbf{3 0}$ days-neuropathic mice. (A) First: IL-17 positive profiles (green fluorescent) in vehicle treated 30 days-neuropathic mice (SNI + vehicle). Second: CD4 positive profiles (red fluorescent) in vehicle treated 30 days-neuropathic mice. Third: double labeling of IL-17 and CD4 positive profiles. Fourth: higher magnification showing that the cells expressing IL-17 were T Iymphocytes. Cell nuclei were counterstained with DAPI (blue fluorescence). Scale bars: $100 \mu \mathrm{m}$. (B) First: IL-1 $\beta$ positive profiles (green fluorescent) in vehicle treated 30 days-neuropathic mice. Second: GFAP positive profiles (red fluorescent) in vehicle treated 30 days-neuropathic mice. Third: double labeling of GFAP and IL-1 $\beta$ positive profiles. Fourth: higher magnification indicating that the cells expressing IL-1 $\beta$ were astrocytes. Cell nuclei were counterstained with DAPI (blue fluorescence) Scale bars: $100 \mu \mathrm{m}$. (C) First: IL-1 $\beta$ profiles (green fluorescent) in hMSC-treated 30 days-neuropathic mice (SNI + hMSCs). Second: IL-17 profiles (green fluorescent) in hMSC-treated 30 days-neuropathic mice (SNI + hMSCs). The absence of positive staining indicates that injected hMSCs were able to reduced the protein levels of pro-inflammatory interleukins IL-1 $\beta$ and IL-17, confirming western blot analysis results.

invasive surgical procedure, as performed in our previous work (Siniscalco et al., 2010), and (ii) following the clinical trial protocols currently used more effectively (see www.clinicaltrials.gov). Indeed, in all clinical use of human adult bone marrow derived, culture-expanded MSCs, whether autologous or allogeneic, no adverse events were recorded. This means that cell isolation and culture expansion is safe and clinical benefits from the intravenous delivery of hMSCs could be observed (Caplan, 2009).

Once hMSCs had been injected into neuropathic mice, we focused our biomolecular and morphological experiments on the L4-L5 spinal cord, since there is evidence that this may be the primary site directly involved in NP-associated cellular and molecular changes (Maione et al., 2002; de Novellis et al., 2004).

\section{HUMAN MESENCHYMAL STEM CELL MECHANISM OF ACTION}

Despite hMSCs having been employed in several clinical trials, their mechanism of action is still unknown. Their main advantage is that they are probably immunomodulatory and versatile in their action due to their secreted bioactive anti-inflammatory and regenerative molecules (Bonfield and Caplan, 2010). Indeed, hMSC-mediated secretion of a broad range of bioactive molecules, such as growth factors, cytokines, and chemokines, is their most biologically significant role under injury conditions rather than their ability to differentiate (Pan et al., 2007; Pisati et al., 2007; Siniscalco et al., 2008, 2010; Meirelles Lda et al., 2009). This fact exceeds the thesis based on differentiation, engraftment, or de novo regeneration from transplanted stem cells to a point of view in which the functional benefit derived from hMSC transplantation may be due to their ability to produce factors that activate endogenous restorative mechanisms. A combination of neurotrophic and anti-inflammatory factors produced by transplanted hMSCs could be the mechanism underlying the functional improvement in NP seen after hMSC injection shown in the present study. Indeed, MSC paracrine effects can be divided into several types: trophic, immunomodulatory, anti-scarring, and chemoattractant. The most surprising feature of MSCs is their extraordinary and still partially unexplained immunomodulatory property (Nauta and Fibbe, 2007; Schipani and Kronenberg, 2009). Mesenchymal SCs have a potent immunosuppressive capacity mediated via soluble factors. These immunomodulatory effects of MSCs consist of the inhibition of the proliferation of CD8+ and CD4+ T lymphocytes and natural killer (NK) cells, suppression of immunoglobulin production by plasma cells, inhibition of maturation of dendritic cells (DCs) and stimulation of the proliferation of regulatory $\mathrm{T}$ cells (Hoogduijn et al., 2010). It has been demonstrated that MSCs are able to inhibit $\mathrm{T}$ lymphocyte proliferation and pro-inflammatory cytokine production after mitogen or cellular stimulation in vitro (Di Nicola et al., 2002; Beyth et al., 2005), as well as in vivo (Dazzi and Marelli-Berg, 2008).

\section{INVOLVEMENT OF THE IMMUNE SYSTEM IN THE NEUROPATHIC PAIN SYNDROME}

The development of NP involves not only neuronal pathways, but also components of the peripheral immune system (Scholz and Woolf, 2007). NP shows a presence of inflammation at the site of the injured nerves. This inflammatory response results in the concentration and activation of innate immune cells at the site of tissue injury. These cells release immunoactive substances, such as cytokines, neurotrophic factors, and chemokines, which initiate local actions and can result in a more generalized immune response (Vallejo et al., 2010). Spinal cord T lymphocytes are responsible for mediating adaptive immune responses and are involved in NP (Zenonos and Kim, 2010). Indeed, NP-activated CD4+ cells are able to produce IL-17, the interleukin responsible for modulating immune response during inflammation. Interestingly, it has been demonstrated that IL-17 could attract and activate macrophages in a NP condition (Kleinschnitz et al., 2006).

Moreover, emerging studies highlight a key role played by macrophages in the pathogenesis of NP (Vega-Avelaira et al., 2009; Mika et al., 2010; Otoshi et al., 2010). In the NP condition, activated macrophages are able to over-express pro-nociceptive mediators, among which the inflammatory protein- $1 \alpha$ (MIP- $1 \alpha)$, which is in turn capable to up-regulate the potent pro-inflammatory/pronociceptive interleukin IL-1 $\beta$ (Wang et al., 2008; Maeda et al., 2009; Kiguchi et al., 2010; Siniscalco et al., 2010).

\section{EVIDENCE OF HUMAN MESENCHYMAL STEM CELL MECHANISM OF ACTION IN NEUROPATHIC PAIN}

It is worth noting that injected hMSCs were able to down-regulate the NP-increased IL-1 $\beta$ protein level in the spinal cord of NP 
mice, together with a reduced protein level of pro-inflammatory interleukin IL-17. This hMSC anti-inflammatory action could be mediated by switched macrophage phenotypes, as confirmed by the up-regulation of CD206 protein level in the spinal cord of NP mice that we found after hMSC administration. Indeed, hMSCs could mediate switching from M1 pro-inflammatory macrophages to M2 anti-inflammatory macrophages (Kim and Hematti, 2009). These M2 cells highly express the cell surface marker CD206 (Porcheray et al., 2005) and are strongly involved in the resolution of inflammation through trophic factor synthesis and intense phagocytic activities. In addition, MSC-educated macrophages are able to over-express anti-inflammatory cytokines, above all IL-10 (Kim and Hematti, 2009). Our results suggest that hMSC-educated macrophages could be the major type of effector cell through which hMSCs might exert their therapeutic effects.

How hMSCs work to switch macrophages is still under investigation. Emerging studies suggest that hMSC immune-modulating properties are mediated by a novel contact-dependent mechanism involving cell-cell interaction (Gur-Wahnon et al., 2009). It has in fact been demonstrated that hMSCs block antigen-presenting cell (APC) maturation in a contact-dependent manner (Gur-Wahnon et al., 2007). The novel contact-dependent mechanism is different from that mediated by the release of soluble factors and cytokines. The beneficial effects mediated by hMSCs shown here may be the result of both mechanisms, suggesting that they have different yet complementary biological functions. In our study, in spinal cord and cortex niches, hMSCs could secrete molecules to communicate with and respond to other cell types, as well as inducing phenotypic macrophage switching through a contact-dependent mechanism. NP progression may be mediated by modifications in glial responses to nociception (de Novellis et al., 2004). It is noteworthy that also NP-activated astrocytes are able to produce IL-1 $\beta$ (Siniscalco et al., 2010). Our results indicate that astrocytes, rather than microglial cells, may contribute to long-lasting NP maintenance. hMSC-mediated reduction in IL-1 $\beta$ levels, consistently with our earlier results (Siniscalco et al., 2010), suggests that hMSCs were also able to affect the functionality of these cell types, probably by influencing the glial-cytokine-neuronal interactions.

\section{HOMING OF HUMAN MESENCHYMAL STEM CELL IN NEUROPATHIC PAIN CONTROLLING AREAS}

Our data indicate that 26 days after injection, injected hMSCs were able to home to CNS areas critically involved in NP signaling: the dorsal and ventral L4-L5 spinal cord and pre-frontal cortex. Driven by chemokine signals, stem cells are highly capable of homing to sites of injury (Corcione et al., 2006; Sze et al., 2007), where they exert a local and/or systemic immunosuppressive action, thus limiting the inflammatory or immune reactions (Ceradini et al., 2004; Forte et al., 2008; Mattsson, 2008; Siniscalco et al., 2010). In light of our results demonstrating a significant reduction in NP after hMSC treatment, the spinal cord and the cortical brain areas may represent critical substrates in which to analyze specific biomarkers involved in NP processing (Jasmin et al., 2003; Zhuo, 2007; Fuccio et al., 2009; Neugebauer et al., 2009; Siniscalco et al., 2010). In order to perform RT-PCR analysis, we used primer pairs designed to be specifically direct to GAPDH human gene. Thus, the human specificity of these PCR primers that we used to track hMSCs allowed us to ensure that stem cells located in these areas involved in NP modulation were heterologous injected stem cells. In addition, and as further confirmation, we successfully stained injected hMSCs with the cyto-specific marker CD73 in both the dorsal and ventral L4-L5 spinal cord. We did not perform a time-course tracking of injected hMSCs during the progression of NP. However, it has already been demonstrated that hMSCs transplanted into the mouse tail vein were mainly found trapped in the lung initially (Gao et al., 2001; Ankrum and Karp, 2010). It is interesting to note that these lung trapped hMSCs are able to secrete antiinflammatory factors (Lee et al., 2009). Such a possibility could also be the case in our study, where a percentage of hMSCs could have been first trapped in the lung, as demonstrated by GAPDH weak band detected by RT-PCR, where they still secreted antiinflammatory factors, after which they were probably removed. Another percentage of injected hMSCs could have been trapped in the spinal cord and cortex areas, where they homed until up to 26 days post-injection, recruited by NP-associated molecular and cellular changes. Indeed, hMSCs are sensitive to site-specific microenvironments. Due to this fact, pro-nociceptive molecules released in these areas following injury-induced changes in their expression were able to recruit hMSCs. Recruited hMSCs were able to integrate into the tissue, survive, and drive functional improvement. In addition, we did not rule out the possibility that hMSCs could increase the expression of several growth factors, such as nerve growth factor (NGF) and brain-derived neurotrophic factor (BDNF; Mahmood et al., 2004). However, further biomolecular experiments on cortex area will be needed.

We cannot exclude the possibility that injected hMSCs survived more than 3 months post-injection. Nevertheless, whether hMSCs are still present or not, what is fundamental is that they determine long-lasting recovery from NP symptoms.

In conclusion, we show here that hMSCs are able to reduce mechanical allodynia, thermal hyperalgesia and modify cellular and molecular NP mechanisms, once systemically administered into the mouse tail vein. We demonstrated that hMSC treatment reduced the protein levels of the pro-inflammatory interleukins IL$1 \beta$ and IL-17 and increased those of anti-inflammatory interleukin IL-10, as well as the macrophage marker CD206. As a possible mechanism of action of hMSCs in reducing NP, we suggest that hMSCs could exert their beneficial action through a restorative mechanism involving: (i) a cell-to-cell contact activation mechanism, through which spinal cord homed hMSCs are responsible for switching pro-inflammatory macrophages to anti-inflammatory macrophages; (ii) the secretion of a broad spectrum of molecules to communicate with other cell types. Our findings on injected hMSC homing to the site of NP processing provide evidence that the administration of hMSC may be a more suitable long-lasting therapy than currently available anti-neuropathic drugs, supporting the hypothesis that hMSCs may deliver bioactive agents in a site-specific manner, with enduring modulation on the immuneneural cell cross-talk in a rather different way from how the current classical drugs work (Bonfield and Caplan, 2010).

\section{ACKNOWLEDGMENTS}

The authors thank Dr. Tiziana Squillaro, Second University of Naples, for her useful assistance in RT-PCR primers development. Financial support from MIUR-Italy (PRIN 2007, recipient Sabatino Maione) is gratefully acknowledged. 


\section{REFERENCES}

Ankrum, J., and Karp, J. M. (2010). Mesenchymal stem cell therapy: two steps forward, one step back. Trends. Mol. Med. 16, 203-209.

Beggs, K. J., Lyubimov, A., Borneman, J. N., Bartholomew, A., Moseley, A., Dodds, R., Archambault, M. P., Smith, A. K., and McIntosh, K. R. (2006). Immunologic consequences of multiple, high-dose administration of allogeneic mesenchymal stem cells to baboons. Cell Transplant. 15, 711-721.

Beyth, S., Borovsky, Z., Mevorach, D., Liebergall, M., Gazit, Z., Aslan, H., Galun, E., and Rachmilewitz, J. (2005). Human mesenchymal stem cells alter antigen-presenting cell maturation and induce $\mathrm{T}$ cell unresponsiveness. Blood 105, 2214-2219.

Bonfield, T. L., and Caplan, A. I. (2010). Adult mesenchymal stem cells: an innovative therapeutic for lung diseases. Discov. Med. 9, 337-345.

Bourquin, A. F., Süveges, M., Pertin, M., Gilliard, N., Sardy, S., Davison, A. C., Spahn, D. R., and Decosterd, I. (2006). Assessment and analysis of mechanical allodynia-like behavior induced by spared nerve injury (SNI) in the mouse. Pain 122, 14.e114.e14.

Bradford, M. M. (1976). A rapid and sensitive method for the quantization of microgram quantities of protein utilizing the principle of protein-dye binding. Anal. Biochem. 72, 248-254.

Cao, Q., Benton, R. L., and Whittemore, S. R. (2002). Stem cell repair of central nervous system injury. $J$. Neurosci. Res. 68, 501-510.

Caplan, A. I. (2009). Why are MSCs therapeutic? New data: new insight. J. Pathol. 217, 318-324.

Ceradini, D. J., Kulkarni, A. R., Callaghan, M. J., Tepper, O. M., Bastidas, N., Kleinman, M. E., Capla, J. M., Galiano, R. D., Levine, J. P., and Gurtner, G. C. (2004). Progenitor cell trafficking is regulated by hypoxic gradients through HIF1 induction of SCF-1. Nat. Med. 10, 858-864.

Corcione, A., Benvenuto, F., Ferretti, E., Giunti, D., Cappiello, V., Cazzanti, F., Risso, M., Gualandi, F., Mancardi, G. L., Pistoia, V., and Uccelli, A. (2006). Human mesenchymal stem cells modulate B-cell functions. Blood 107, 367-372.

Dazzi, F., and Marelli-Berg, F. M. (2008). Mesenchymal stem cells for graftversus-host disease: close encounters with T cells. Eur. J. Immunol. 38, 1479-1482. de Novellis, V., Siniscalco, D., Galderisi, U., Fuccio, C., Nolano, M., Santoro, L., Cascino, A., Roth, K. A., Rossi, F., and Maione, S. (2004). Blockade of glutamate mGlu5 receptors in a rat model of neuropathic pain prevents early over-expression of pro-apoptotic genes and morphological changes in dorsal horn lamina II. Neuropharmacology 46, 468-479.

Di Nicola, M., Carlo-Stella, C., Magni, M., Milanesi, M., Longoni, P. D., Matteucci, P., Grisanti, S., and Gianni, A. M. (2002). Human bone marrow stromal cells suppress $\mathrm{T}$ lymphocyte proliferation induced by cellular or nonspecific mitogenic stimuli. Blood 99, 3838-3843.

Forte, A., Finicelli, M., Mattia, M., Berrino, L., Rossi, F., De Feo, M., Cotrufo, M., Cipollaro, M., Cascino, A., and Galderisi, U. (2008). Mesenchymal stem cells effectively reduce surgically induced stenosis in rat carotids. J. Cell. Physiol. 217, 789-799.

Fuccio, C., Luongo, C., Capodanno, P., Giordano, C., Scafuro, M. A., Siniscalco, D., Lettieri, B., Rossi, F., Maione, S., and Berrino, L. (2009). A single subcutaneous injection of ozone prevents allodynia and decreases the over-expression of pro-inflammatory caspases in the orbito-frontal cortex of neuropathic mice. Eur. J. Pharmacol. 603, 42-49.

Galluzi, K. E. (2005). Management of neuropathic pain. J. Am. Osteopath. Assoc. 105, 12-19.

Gao, J., Dennis, J. E., Muzic, R. F., Lundberg, M., and Caplan, A. I. (2001). The dynamic in vivo distribution of bone marrow-derived mesenchymal stem cells after infusion. Cells Tissues Organs (Print) 169, 12-20.

Giordano, A., Galderisi, U., and Marino, I. R. (2007). From the laboratory bench to the patient's bedside: an update on clinical trials with mesenchymal stem cells. J. Cell. Physiol. 211, 27-35.

Gur-Wahnon, D., Borovsky, Z., Beyth, S., Liebergall, M., and Rachmilewitz, J. (2007). Contact-dependent induction of regulatory antigenpresenting cells by human mesenchymal stem cells is mediated via STAT3 signaling. Exp. Hematol. 35, 426-433.

Gur-Wahnon, D., Borovsky, Z., Liebergall, M., and Rachmilewitz, J. (2009). The induction of APC with a distinct tolerogenic phenotype via contactdependent STAT3 activation. PLoS ONE 4, e6846. doi:10.1371/journal.pone.0006846

Hoogduijn, M. J., Popp, F., Verbeek, R., Masoodi, M., Nicolaou, A., Baan,
C., and Dahlke, M. H. (2010). The immunomodulatory properties of mesenchymal stem cells and their use for immunotherapy. Int. Immunopharmacol. 10, 1496-1500.

Jasmin, L., Rabkin, S. D., Granato, A., Boudah, A., and Ohara, P. T. (2003). Analgesia and hyperalgesia from GABA-mediated modulation of the cerebral cortex. Nature 424 316-320.

Kiguchi, N., Maeda, T., Kobayashi, Y., Fukazawa, Y., and Kishioka, S. (2010). Macrophage inflammatory protein-1alpha mediates the development of neuropathic pain following peripheral nerve injury through interleukin-1beta up-regulation. Pain 149, 305-315.

Kim, J., and Hematti, P. (2009). Mesenchymal stem cell-educated macrophages: a novel type of alternatively activated macrophages. Exp. Hematol. 37, 1445-1453.

Klass, M., Gavrikov, V., Drury, D. Stewart, B., Hunter, S., Denson, D. D., Hord, A., and Csete, M. (2007). Intravenous mononuclear marrow cells reverse neuropathic pain from experimental mononeuropathy. Anesth. Analg. 104, 944-948.

Kleinschnitz, C., Hofstetter, H. H. Meuth, S. G., Braeuninger, S., Sommer, C., and Stoll, G. (2006). T cell infiltration after chronic constriction injury of mouse sciatic nerve is associated with interleukin17 expression. Exp. Neurol. 200, 480-485.

Le Blanc, K., and Pittenger, M. (2005). Mesenchymal stem cells: progress toward promise. Cytotherapy 7, 36-45.

Lee, R. H., Pulin, A. A., Seo, M. J., Kota, D. J., Ylostalo, J., Larson, B. L., Semprun-Prieto, L., Delafontaine, P., and Prockop, D. J. (2009). Intravenous hMSCs improve myocardial infarction in mice because cells embolized in lung are activated to secrete the anti-inflammatory protein TSG-6. Cell Stem Cell 5, 54-63.

Lindvall, O., and Kokaia, Z. (2005). Stem cell therapy for human brain disorders. Kidney Int. 68, 1937-1939.

Lindvall, O., and Kokaia, Z. (2006). Stem cells for the treatment of neurological disorders. Nature 441, 1094-1096.

Maeda, T., Kiguchi, N., Kobayashi, Y., Ikuta, T., Ozaki, M., and Kishioka, S. (2009). Leptin derived from adipocytes in injured peripheral nerves facilitates development of neuropathic pain via macrophage stimulation. Proc. Natl. Acad. Sci. U.S.A. 106, 13076-13081.
Mahmood, A., Lu, D., and Chopp, M. (2004). Intravenous administration of marrow stromal cells (MSCs) increases the expression of growth factors in rat brain after traumatic brain injury. J. Neurotrauma 21, 33-39.

Maione, S., Siniscalco, D., Galderisi, U., de Novellis, V., Uliano, R., Di Bernardo, G., Berrino, L., Cascino, A., and Rossi, F. (2002). Apoptotic genes expression in the lumbar dorsal horn in a model neuropathic pain in rat. Neuroreport 13, 101-106.

Mattsson, J. (2008). Recent progress in allogeneic stem cell transplantation. Curr. Opin. Mol. Ther. 10, 343-349.

Meirelles Lda, S., Fontes, A. M., Covas, D. T., and Caplan, A. I. (2009). Mechanisms involved in the therapeutic properties of mesenchymal stem cells. Cytokine Growth Factor Rev. 20, 419-427.

Merskey, H., and Bogduk, N. (1994). Classification of Chronic Pain. Seattle: IASP Press.

Mika, J., Rojewska, E., Makuch, W., and Przewlocka, B. (2010). Minocycline reduces the injury-induced expression of prodynorphin and pronociceptin in the dorsal root ganglion in a rat model of neuropathic pain. Neuroscience 165, 1420-1428.

Musolino, P. L., Coronel, M. F., Hokfelt, T., and Villar, M. J. (2007). Bone marrow stromal cells induce changes in pain behavior after sciatic nerve constriction. Neurosci. Lett. 418, 97-101.

Nauta, A. J., and Fibbe, W. E. (2007). Immunomodulatory properties of mesenchymal stromal cells. Blood 110, 3499-3506.

Neugebauer, V., Galhardo, V., Maione, S., and Mackey, S. C. (2009). Forebrain pain mechanisms. Brain Res. Rev. 60, 226-242.

Otoshi, K., Kikuchi, S., Konno, S., and Sekiguchi, M. (2010). The reactions of glial cells and endoneurial macrophages in the dorsal root ganglion and their contribution to pain-related behavior after application of nucleus pulposus onto the nerve root in rats. Spine 35, 10-17.

Pan, H. C., Cheng, F. C., Chen, C. J., Lai, S. Z., Lee, C. W., Yang, D. Y., Chang, M. H., and Ho, S. P. (2007). Post-injury regeneration in rat sciatic nerve facilitated by neurotrophic factors secreted by amniotic fluid mesenchymal stem cells. J. Clin. Neurosci. 14, 1089-1098.

Petrie Aronin, C. E., and Tuan, R. S. (2010). Therapeutic potential of the immunomodulatory activities of adult mesenchymal stem cells. Birth Defects Res. C Embryo Today 90, 67-74. 
Pisati, F., Bossolasco, P., Meregalli, M., Cova, L., Belicchi, M., Gavina, M., Marchesi, C., Calzarossa, C., Soligo, D., Lambertenghi-Deliliers, G., Bresolin, N., Silani, V., Torrente, Y., and Polli, E. (2007). Induction of neurotrophin expression via human adult mesenchymal stem cells: implication for cell therapy in neurodegenerative diseases. Cell Transplant. 16, 41-55.

Porcheray, F., Viaud, S., Rimaniol, A. C., Léone, C., Samah, B., DereuddreBosquet, N., Dormont, D., and Gras, G. (2005). Macrophage activation switching: an asset for the resolution of inflammation. Clin. Exp. Immunol. 142, 481-489.

Pricola, K. L., Kuhn, N. Z., HaleemSmith, H., Song, Y., and Tuan, R. S. (2009). Interleukin-6 maintains bone marrow-derived mesenchymal stem cell stemness by an ERK1/2-dependent mechanism. J. Cell. Biochem. 108, 577-588.

Sarugaser, R., Lickorish, D., Baksh, D., Hosseini, M. M., and Davies, J. E. (2005). Human umbilical cord perivascular (HUCPV) cells: a source of mesenchymal progenitors. Stem Cells 23, 220-229.

Schipani, E., and Kronenberg, $\mathrm{H}$. M. (2009). "Adult mesenchymal stem cells," in StemBook, Edn. The Stem Cell Research Community, Eds K. R. Chien, (Cambridge,
MA: Harvard Stem Cell Institute), doi/10.3824/stembook.1.38.1

Scholz, J., and Woolf, C. J. (2007). The neuropathic pain triad: neurons, immune cells and glia. Nat. Neurosci. 10, 1361-1368.

Siniscalco, D. (2010). Transplantation of human mesenchymal stem cells in the study of neuropathic pain. Methods Mol. Biol. 617, 337-345.

Siniscalco, D., de Novellis, V., Rossi, F., and Maione, S. (2005a). Neuropathic pain: is the end of suffering starting in the gene therapy? Curr. Drug Targets 6, 75-80.

Siniscalco, D., Fuccio, C., de Novellis, V., Rossi, F., and Maione, S. (2005b). Molecular methods for neuropathic pain treatment. J. Neuropathic Pain 1, 35-42.

Siniscalco, D., Giordano, C., Galderisi, U., Luongo, L., Alessio, N., Di Bernardo, G., de Novellis, V., Rossi, F., and Maione, S. (2010). Intrabrain microinjection of human mesenchymal stem cells decreases allodynia in neuropathic mice. Cell. Mol. Life Sci. 67, 655-669.

Siniscalco, D., Rossi, F., and Maione, S. (2007). Molecular approaches for neuropathic pain treatment. Curr. Med. Chem. 14, 1783-1787.

Siniscalco, D., Rossi, F., and Maione, S. (2008). Stem cell therapy for neuropathic pain treatment. J. Stem Cells Regen. Med. 3, 2-11.
Sze, S. K., de Kleijn, D. P., Lai, R. C. Tan, E. K., Zhao, H., Yeo, K. S., Low, T. Y., Lian, Q., Lee, C. N., Mitchell, W., El Oakley, R. M., and Lim, S. K. (2007). Elucidating the secretion proteome of human embryonic stem cell-derived mesenchymal stem cells. Mol. Cell Proteomics 6, 1680-1689.

Vallejo, R., Tilley, D. M., Vogel, L., and Benyamin, R. (2010). The role of glia and the immune system in the development and maintenance of neuropathic pain. Pain Pract. 10, 167-184.

Vega-Avelaira, D., Géranton, S., and Fitzgerald, M. (2009). Differential regulation of immune responses and macrophage/neuron interactions in the dorsal root ganglion in young and adult rats following nerve injury. Mol. Pain 5, 70.

Wang, Z., Wang, J., Li, X., Yuan, Y. and Fan, G. (2008). Interleukin-1 beta of Red nucleus involved in the development of allodynia in spared nerve injury rats. Exp. Brain Res. 188, 379-384.

Zenonos, G., and Kim, J. E. (2010). A T cell-orchestrated immune response in the adult dorsal spinal cord as a cause of neuropathic painlike hypersensitivity after peripheral nerve damage: a door to novel therapies? Neurosurgery 66 N24-N25.

Zhuo, M. (2007). Neuronal mechanism for neuropathic pain. Mol. Pain 3, 14.
Zuk, P. A., Zhu, M., Ashjian, P., De Ugarte, D. A., Huang, J. I., Mizuno, H., Alfonso, Z. C., Fraser, J. K., Benhaim, P., and Hedrick, M. H. (2002). Human adipose tissue is a source of multipotent stem cells. Mol. Biol. Cell 13, 4279-4295.

Conflict of Interest Statement: The authors declare that the research was conducted in the absence of any commercial or financial relationships that could be construed as a potential conflict of interest.

Received: 12 September 2011; accepted: 16 November 2011; published online: 01 December 2011.

Citation: Siniscalco D, Giordano $C$, Galderisi $U$, Luongo $L$, de Novellis $V$, Rossi F and Maione S (2011) Longlasting effects of human mesenchymal stem cell systemic administration on pain-like behaviors, cellular, and biomolecular modifications in neuropathic mice. Front. Integr. Neurosci. 5:79. doi: 10.3389/fnint.2011.00079

Copyright (c) 2011 Siniscalco, Giordano, Galderisi, Luongo, de Novellis, Rossi and Maione. This is an open-access article distributed under the terms of the Creative Commons Attribution Non Commercial License, which permits noncommercial use, distribution, and reproduction in other forums, provided the original authors and source are credited. 